\title{
High prevalence of biofilm synergy among bacterial soil isolates in cocultures indicates bacterial interspecific cooperation
}

\author{
Dawei Ren, Jonas S Madsen, Søren J Sørensen and Mette Burmølle \\ Section of Microbiology, Department of Biology, University of Copenhagen, Copenhagen, Denmark
}

\begin{abstract}
Biofilms that form on roots, litter and soil particles typically contain multiple bacterial species. Currently, little is known about multispecies biofilm interactions and few studies have been based on environmental isolates. Here, the prevalence of synergistic effects in biofilm formation among seven different soil isolates, cocultured in combinations of four species, was investigated. We observed greater biofilm biomass production in $63 \%$ of the four-species culture combinations tested than in biofilm formed by single-species cultures, demonstrating a high prevalence of synergism in multispecies biofilm formation. One four-species consortium, composed of Stenotrophomonas rhizophila, Xanthomonas retroflexus, Microbacterium oxydans and Paenibacillus amylolyticus, exhibited strong synergy in biofilm formation and was selected for further study. Of the four strains, $X$. retroflexus was the only one capable of forming abundant biofilm in isolation, under the in vitro conditions investigated. In accordance, strain-specific quantitative PCR revealed that $X$. retroflexus was predominant within the four-species consortium ( $>97 \%$ of total biofilm cell number). Despite low relative abundance of all the remaining strains, all were indispensable for the strong synergistic effect to occur within the four-species biofilm. Moreover, absolute individual strain cell numbers were significantly enhanced when compared with those of single-species biofilms, indicating that all the individual strains benefit from inclusion in the multispecies community. Our results show a high prevalence of synergy in biofilm formation in multispecies consortia isolated from a natural bacterial habitat and suggest that interspecific cooperation occurs.
\end{abstract}

The ISME Journal (2015) 9, 81-89; doi:10.1038/ismej.2014.96; published online 17 June 2014

\section{Introduction}

Environmental biofilm systems are comprised of many diverse cohabiting bacteria (Hall-Stoodley et al., 2004). For example, soil is typically high in bacterial abundance and diversity and includes readily available surfaces suggesting it is a good setting for multispecies biofilm formation (Burmølle et al., 2012). Members of multispecies biofilms may influence each other antagonistically; for example, through resource competition or production of inhibitory compounds (Rao et al., 2005), or synergistically; via mechanisms such as syntrophy (cometabolim), biofilm induction or enhanced resistance (Burmølle et al., 2014). We have previously observed strong synergy among four epiphytic isolates from a marine environment. When comparing single-species biofilms with four-species biofilms, enhanced biofilm biomass was observed in the latter, in addition to higher resistance to antimicrobial agents and better protection from

Correspondence: M Burmølle or SJ Sørensen, Universitetsparken 15, Bygning. 1, Copenhagen 2100, Denmark.

E-mail: burmolle@bio.ku.dk or sjs@bio.ku.dk

Received 20 December 2013; revised 8 May 2014; accepted 10 May 2014; published online 17 June 2014 bacterial invasion (Burmølle et al., 2006). Likewise, Diaz et al. (2012) revealed a synergistic partnership between Candida albicans and streptococci in which $C$. albicans improved the ability of streptococci to form biofilms on abiotic surfaces and on an oral mucosa analog (Diaz et al., 2012). In addition, several studies have shown that some species that are unable to form biofilms in isolation can promote mixed-species biofilm biomass (Filoche et al., 2004; Sharma et al., 2005; Yamada et al., 2005; Klayman et al., 2009). In a recent study, Lee et al. (2014) addressed the protective effect of the resistant species in a three-species biofilm. These resistant species protected the more sensitive ones from inhibitory compounds and the overall species ratio remained constant, implying that resistance mechanisms may serve as public goods in multispecies biofilms (Lee et al., 2014). These examples are all indicative of synergistic effects in multispecies biofilm formation, but the prevalence and impact of these effects among natural bacterial isolates has, to the best of out knowledge, not yet been addressed.

Multispecies biofilms have an essential role in maintaining the ecological balance in soil (Burmølle et al., 2012) and, compared with single-species biofilm or planktonic counterparts, seem to confer 
benefits such as increased resistance to antibacterial compounds, enhanced protection from desiccation and protozoan predation, and elevated rates of horizontal gene transfer (Davey and Otoole, 2000; Jefferson, 2004; Sørensen et al., 2005). Therefore, a pressing need exists for research directed toward understanding social interactions and selective forces that drive bacterial biofilm communities. Specifically, the gains/losses in biofilm biomass production and the protection level afforded to each strain in a mixed community must be evaluated, enabling identification of the underlying interactions as cooperative or competitive. In the present study, we define a synergistic effect as an increase in the amount of biofilm biomass produced in cocultures in comparison with that produced by the best biofilm former in monoculture. When such synergistic biofilm enhancement leads to an increase in cell numbers of all strains present in the biofilm, we interpret this as a cooperative trait.

Previous studies have primarily focused on describing synergistic biofilm interactions based on defined, pairwise interactions. Here we assess the prevalence of synergism in four-species biofilm formation in order to determine whether synergistic effects are frequent or rare among the strain collection tested. Different bacterial soil isolates were obtained from one specific soil environment simultaneously. These isolates are therefore presumed to have coexisted. One selected four-species biofilm community was investigated for the presence and progression dynamics of the individual strains at different stages of biofilm development by use of strain-specific quantitative PCR (qPCR). We demonstrate a high prevalence of synergistic effects in multispecies biofilm formation, and enhancement in cell numbers of all four species in the selected multispecies biofilm, indicating that cooperative forces shape these communities.

\section{Materials and methods}

Bacterial strains used in this study

Seven agricultural, bacterial isolates, previously identified and characterized, were used in this study: (1) Pseudomonas lutea, (2) Stenotrophomonas rhizophila, (3) Xanthomonas retroflexus, (4) Ochrobactrum rhizosphaerae, (5) Microbacterium oxydans, (6) Arthrobacter nitroguajacolicus and (7) Paenibacillus amylolyticus (de la Cruz Perera et al., 2013; Ren et al., 2013).

Biofilm quantification by use of crystal violet assay Biofilm formation was assayed and quantified as previously described (Ren et al., 2013). Briefly, exponential phase cultures of the seven selected strains were adjusted to an optical density at $590 \mathrm{~nm}$ $\left(\mathrm{OD}_{590}\right)$ of 0.15 in tryptic soy broth (Merck KgaA, Darmstadt, Germany) medium and then inoculated into Nunc-TSP plate (Cat. No. 445497, Thermo Scientific, Hvidovre, Denmark). The inoculum volumes were $160 \mu \mathrm{l}$ for monospecies biofilms and $40 \mu \mathrm{l}$ for each species in four-species biofilms. After 24-h incubation at $24{ }^{\circ} \mathrm{C}$ with shaking (200 r.p.m.), biofilm formation was quantified by a modified crystal violet (CV) assay based on the Calgary device (Ceri et al., 1999; Ren et al., 2013).

The four-species consortium composed of strains 2, 3, 5 and 7 was examined for synergy in all possible combinations of two-, three- and fourspecies biofilm. The inoculum volumes of each strain were equivalent and added up to a total of $160 \mu \mathrm{l}$. Biofilm assays were performed as described above.

The biofilm experiments were repeated three times on three independent days with four replicates each time. The statistical analyses were conducted using analysis of variance test (SPSS version 17.0 for Windows; SPSS Inc., Chicago, IL, USA). $P$-values $<0.05$ were regarded as statistically significant.

Effects of spent biofilm culture supernatants on biofilm formation

Single-species biofilms of strains 2, 3, 5 and 7 were prepared as described above. Supernatants were generated by filtering planktonic fractions through $0.2-\mu \mathrm{m}$ pore-size filters. No viable bacteria were detected in the supernatants. Cultures of strains 2, 3, 5 and 7 were grown to exponential phase and then adjusted to an $\mathrm{OD}_{590}$ of 0.3 in $2 \times$ tryptic soy broth media. The four strains were then mixed in equal volumes in all possible combinations of three. Subsequently, $80 \mu \mathrm{l}$ of each of the mixtures was spiked with $80 \mu \mathrm{l}$ supernatant derived from the fourth species. Comparative controls were done by adding $80 \mu \mathrm{l}$ of sterile water instead of supernatant to the three member cocultures. Next, the cocultures were inoculated in wells of microtiter plates in four replicates and incubated at $24^{\circ} \mathrm{C}$ for $24 \mathrm{~h}$. Biofilms formed on the pegs were quantified by using the $\mathrm{CV}$ assay as described above.

\section{Strain-specific qPCR on biofilm and planktonic fractions}

Cell numbers of monocultures and cocultures of strain 2, 3, 5 and 7 were assessed by qPCR as follows: the four-species biofilms attached to the pegs and planktonic cells in wells were collected at six time points $(4 \mathrm{~h}, 8 \mathrm{~h}, 12 \mathrm{~h}, 16 \mathrm{~h}, 20 \mathrm{~h}$ and $24 \mathrm{~h}$ after coinoculation). In addition, both single-species biofilms and associated planktonic fractions were sampled at $24 \mathrm{~h}$. Three replicates were prepared at each time point. Cells were lysed by lysozyme digestion and bead beating, followed by DNA extraction using FastDNA SPIN Kit for soil (Qbiogene, Illkirch, France) and species-specific qPCRs as previously reported (Ren et al., 2013). 
The PCR programs were adjusted as follows: $95{ }^{\circ} \mathrm{C}$ for $2 \mathrm{~min}, 40$ cycles of $95^{\circ} \mathrm{C}$ for $15 \mathrm{~s}, 64^{\circ} \mathrm{C}$ for $20 \mathrm{~s}$ and $72{ }^{\circ} \mathrm{C}$ for $20 \mathrm{~s}$, followed by a standard melting/ dissociation curve segment. Each sample was assayed in duplicate wells and a no-template control was included in every run. As the exact copy numbers of the $16 \mathrm{~S}$ rRNA gene per cell in the four species are currently unknown, the cell numbers were calculated based on other species in the same genus as previously described (Ren et al., 2013).

\section{Results}

Biofilm formation by single-species and four-species consortia

The prevalence of synergistic effects in biofilm formation among the seven soil isolates was examined by coculturing all possible combinations of four strains. A total of 35 different four-species consortia was screened for biofilm formation by using the Nunc-TSP lid system and quantified by the modified CV-staining method, previously demonstrated to be suitable and reproducible (Ren et al., 2013).

For assessment of whether synergistic effects dominated in the individual four-species biofilm, the measured absorbance of the multispecies biofilm $\left(\mathrm{Abs}_{590} \mathrm{MS}\right)$ was related to that of the best singlespecies biofilm former present in the relevant combination $\left(\mathrm{Abs}_{590} \mathrm{BS}\right)$ as follows: $\left(\mathrm{Abs}_{590} \mathrm{MS}-\right.$ standard deviation, s.d. $)>\left(A_{A^{590}} B S+\right.$ s.d. $)=$ synergism.

This is based on the assumption that in the absence of interactions: (1) cell density of singlespecies biofilms and multispecies biofilms is equal, so no additional biofilm is formed by multiple species cultures than by single-species cultures when similar nutrients are available unless interactions causing synergistic effects occur, and (2) the best biofilm former dominates the biofilm. Figure 1 shows data from a representative data set. Data from the three biological replicates are presented as Supplementary material (Supplementary Table S1).

A total of 22 four-species-combined cultures, accounting for $63 \%$ of all combined cultures (35 in total), showed synergy in biofilm formation, as indicated by enhanced biomass production when compared with that of single-species cultures (Figure 1). In total, 10 of the 22 combined cultures showing synergy were composed of only poor biofilm-forming strains. The synergistic effect was especially strong in combinations 1-2-5-7 and 1-2-6-7, in which biofilm biomass had increased by more than fivefold $(P<0.05)$ (Figure $1 \mathrm{~b}$, Supplementary Table S1). This may indicate that interspecific interactions had led to cooperative biofilm formation by these strains despite an inability to form biofilms individually.

The majority of the 13 combinations that were not identified as interacting synergistically with respect to biofilm formation produced amounts of biofilm that were equal to the best biofilm former (Figures 1a and b) with two exceptions. Combinations 1-2-3-4 and 2-3-4-5 showed four-species biofilm formation plus standard deviation (Abs ${ }_{590} \mathrm{MS}+$ s.d.), which was lower than monospecies biofilm formation (best strain) minus standard deviation $\left(\mathrm{Abs}_{590} \mathrm{BS}-\right.$ s.d.). In these two cocultures, competitive interactions may dominate.

Synergy in biofilm formation among S. rhizophila, $X$. retroflexus, $M$. oxydans and $P$. amylolyticus An example of strong synergy was found in strain combination 2-3-5-7 (S. rhizophila, X. retroflexus, $M$. oxydans and $P$. amylolyticus, respectively, Figure 1 and Supplementary Table S1). To determine whether all four strains contributed to the enhanced biomass, each strain was grown as a single-species biofilm and in all possible combinations as two, three and four-species biofilms and analyzed for synergy in biofilm formation as described above (Figure 2).

Two and three-species biofilms did not differ significantly $(P>0.05)$, with the exception of combinations 3-5 $(P=0.026)$ and $3-5-7 \quad(P=0.001)$, when compared with the amount of biofilm produced by single-species cultures. Strain 2, $S$. rhizophila and strain $7, P$. amylolyticus were very weak biofilm-formers when grown alone, but both stimulated biofilm formation of the other three species. When the four isolates were cocultured, the biomass increased by more than fourfold compared with that of single-species biofilms and two-fivefold compared with that of three-species biofilms. This illustrates that each of these four strains were essential for strong synergy induction. Interestingly, this applied both to the good biofilm former, strain $3, X$. retroflexus, as well as the three other strains that only produce very small amounts of biofilm in monocultures.

No significant differences were observed $(P>0.05)$ between three-species biofilms with and without spent, cell-free supernatant. This indicates that the presence of the fourth species was required in all combinations for the synergistic effect to occur, or alternatively that compounds in the supernatant were only transiently effective during the $24 \mathrm{~h}$ of biofilm formation.

\section{Cell numbers in monocultures and cocultures}

Specific qPCRs were performed in order to evaluate the total cell numbers of each strain in both biofilms and planktonic communities at different stages of multispecies biofilm development. Additionally, the cell numbers in single-species biofilms and planktonic cells at $24 \mathrm{~h}$ were measured. The DNA samples from different time points were diluted appropriately in order to yield results within the dynamic range of standard curves generated using 

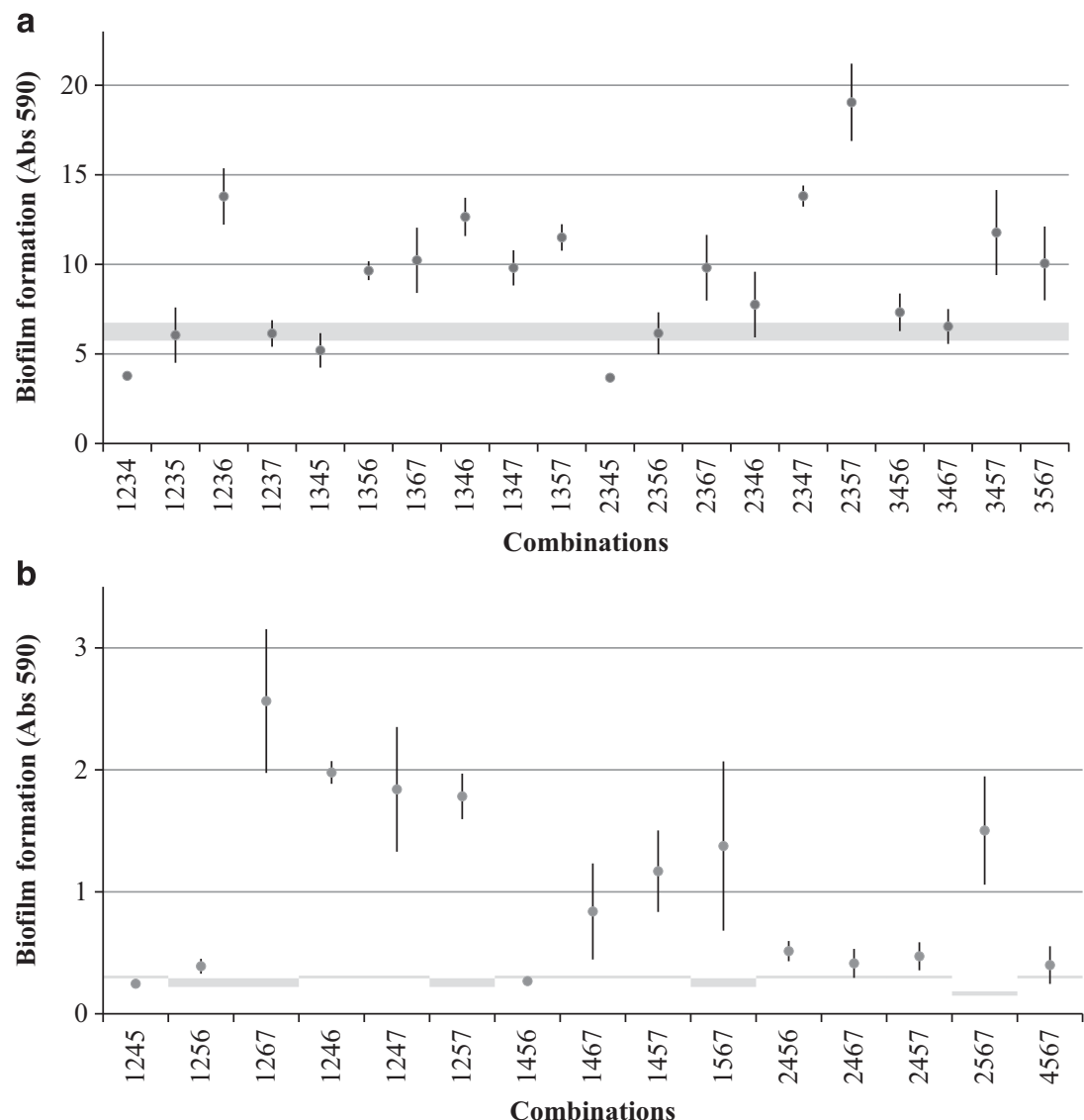

Figure 1 Four-species biofilm formation of seven soil isolates. The observed data points (dark-gray dots) were collected by quantifying multispecies biofilm formation of all possible four-species cocultures using the CV assay. Error bars represent standard deviations of four replicates. Gray bars indicate the amount of monospecies biofilm produced by the best biofilm former present in the specific combination. Data points (including standard deviations) above gray areas indicate synergistic effects in four-species biofilms (see text for further details). The data obtained from combinations containing strain 3 are shown in a, whereas other combinations (without strain 3) are shown in b in order to visualize the data points at the lower end of the range. Strains: 1: P. lutea, 2: S. rhizophila, 3: X. retroflexus, 4: O. rhizosphaerae, 5: M. oxydans, 6: A. nitroguajacolicus, 7: P. amylolyticus.

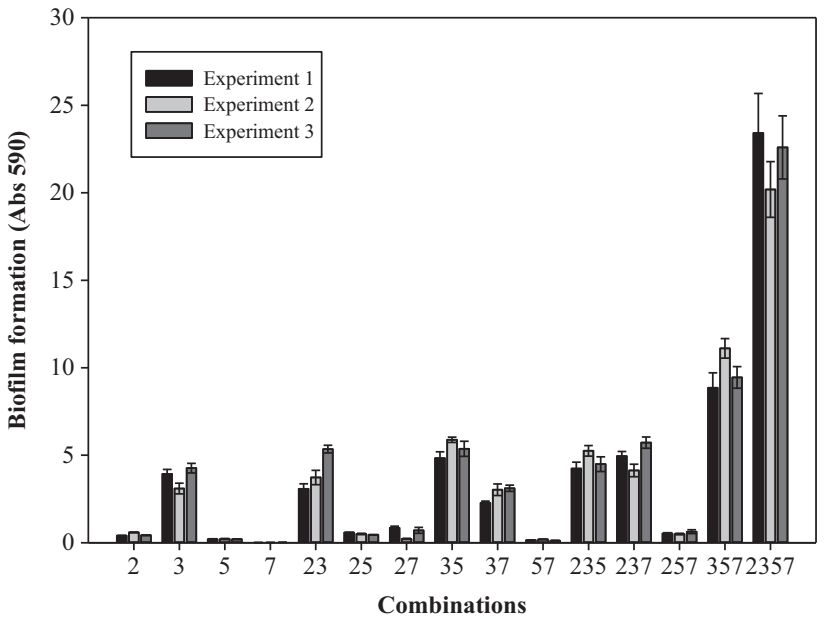

Figure 2 Biofilm formation of four selected strains in monocultures and cocultures. Biofilms formed by four isolates 2: S. rhizophila, 3: X. retroflexus, 5: $M$. oxydans and 7: P. amylolyticus when equal aliquots of the diluted cultures were incubated in cocultures of two, three and four isolates. Assays for the detection of synergistic effects were performed three times (experiments 1,2 and 3) with four replicates each time. Error bars represent \pm s.e.m. of four replicates. duplicate 10-fold dilutions of purified plasmid DNA (Ren et al., 2013). R-square values, based on threshold cycles of the standard curves, ranged from 0.988 to 1 and amplification efficiencies $(E)$ ranged from $84.7-98.0 \%$ (Supplementary Table S2).

The cell numbers of each species in the multispecies biofilm (microtiter peg) and in the associated planktonic fraction (microtiter well) are shown in Figure 3. In general, a marked increase in cell numbers was observed from $4-12 \mathrm{~h}$ for all of the four strains in the biofilm fraction. After $12 \mathrm{~h}$, only the cell numbers of strain 3 increased continuously, whereas those of the other three species in the multispecies biofilm remained constant or decreased. Cell numbers of all four species in the planktonic fraction increased during the first 12-16 h, after which planktonic growth leveled off, indicating nutrient depletion and transition to a steady-state condition.

The cell numbers of each strain in the multispecies biofilm and in the single-species biofilms at $24 \mathrm{~h}$ are shown in Figure 4 . For all of the 

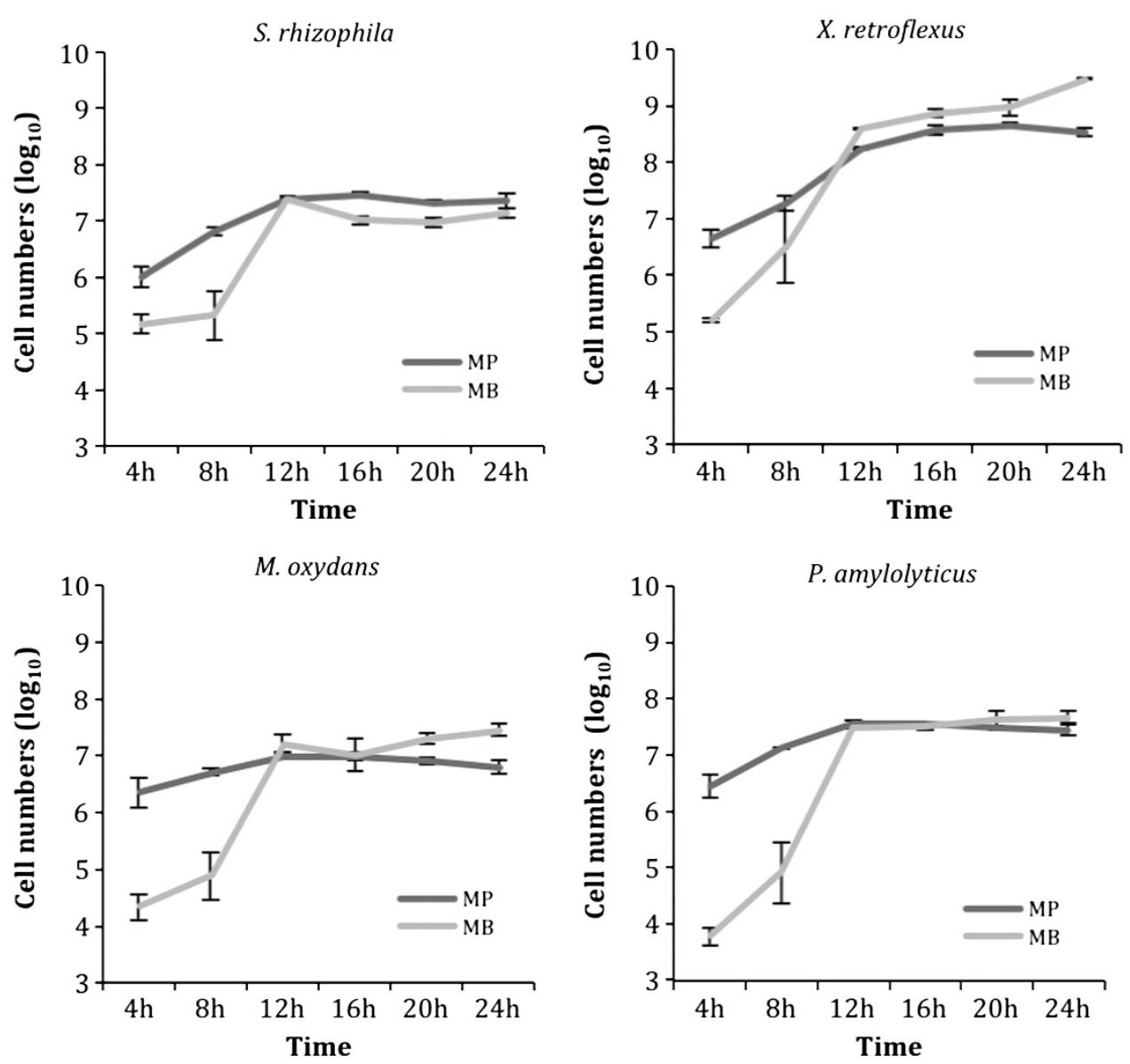

Figure 3 Cell numbers of biofilm (light gray lines) and planktonic (dark gray lines) fraction of each strain in four-species cocultures. The estimated cell numbers per peg or well, respectively, of each strain in four-species biofilms and associated planktonic fractions of S. rhizophila, X. retroflexus, M. oxydans and P. amylolyticus at six time points ( $4 \mathrm{~h}, 8 \mathrm{~h}, 12 \mathrm{~h}, 16 \mathrm{~h}, 20 \mathrm{~h}$ and $24 \mathrm{~h}$ after coincubation) based on qPCR. MP, multispecies planktonic cells; MB, multispecies biofilms. Each point represents the mean of three replicates, with vertical lines representing \pm s.d.

four species, cell numbers were significantly higher $(P<0.05)$ in four-species biofilms in comparison to single-species biofilms, indicative of individual fitness gains from joining the multispecies biofilm.

The ratio of total cell numbers at $24 \mathrm{~h}$ of the four strains (S. rhizophila, X. retroflexus, M. oxydans and P. amylolyticus) was 4:900:9:15 in multispecies biofilms (Table 1). Thus, there was a strong dominance of $X$. retroflexus in the four-species biofilm, as this strain constituted more than $97 \%$ of the total cell number. It is noteworthy that, in contrast to biofilms (Figure 4), planktonic cell numbers of the four species were within equivalent ranges or lower in the four-species coculture than in single-species planktonic biomass (Table 1). The latter was especially pronounced for $M$. oxydans, where a $\log 2$-fold reduction was observed when comparing planktonic cell numbers of this strain in coculture with that in monoculture $\left(6.42 \times 10^{6}-\right.$ $\left.1.58 \times 10^{8}\right)$. The summarized cell numbers from the biofilm and the planktonic fraction from a specific microtiter well are not representative of the total cell number of that well, because there are other surfaces

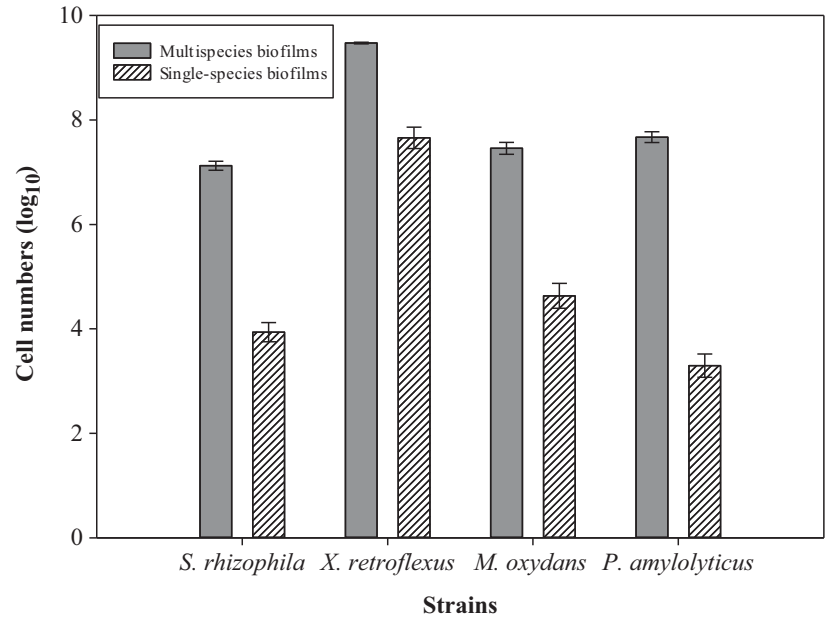

Figure 4 Cell numbers of each strain in single and multi species biofilms. The estimated cell numbers of each strain (S. rhizophila, $X$. retroflexus, $M$. oxydans and P. amylolyticus) in multispecies (gray bars) and single-species (striped bars) biofilms at $24 \mathrm{~h}$. Bars represent means \pm s.d. for three replicates. 
Table 1 Absolute cell numbers per peg/well measured by quantitative PCRs at $24 \mathrm{~h}$ in multispecies planktonic cells, single-species planktonic cells, multispecies biofilms and singlespecies biofilms, and each value is the mean of three replicates

\begin{tabular}{lcccc}
\hline Strains & $\begin{array}{c}\text { Multispecies } \\
\text { planktonic } \\
\text { cells }\end{array}$ & $\begin{array}{c}\text { Single- } \\
\text { species } \\
\text { planktonic } \\
\text { cells }\end{array}$ & $\begin{array}{c}\text { Multispecies } \\
\text { biofilms }\end{array}$ & $\begin{array}{c}\text { Single- } \\
\text { species } \\
\text { biofilms }\end{array}$ \\
\hline S. rhizophila & $2.33 \mathrm{E}+07$ & $5.79 \mathrm{E}+07$ & $1.35 \mathrm{E}+07$ & $9.14 \mathrm{E}+03$ \\
X. retroflexus & $3.38 \mathrm{E}+08$ & $5.46 \mathrm{E}+08$ & $2.97 \mathrm{E}+09$ & $4.92 \mathrm{E}+07$ \\
M. oxydans & $6.42 \mathrm{E}+06$ & $1.58 \mathrm{E}+08$ & $2.93 \mathrm{E}+07$ & $4.73 \mathrm{E}+04$ \\
P. amylolyticus & $2.81 \mathrm{E}+07$ & $1.33 \mathrm{E}+07$ & $4.78 \mathrm{E}+07$ & $2.10 \mathrm{E}+03$ \\
\hline
\end{tabular}

available for bacterial attachment in these wells besides the pegs.

\section{Discussion}

High prevalence of synergism in biofilm formation Single-species biofilms are rare in natural environments; especially in agricultural soil where microcommunities exposed to organic matter have the potential to develop into multispecies biofilms with high bacterial density and diversity (Rodríguez and Bishop, 2007; Narisawa et al., 2008; Burmølle et al., 2010). Such conditions in bacterial habitats are likely to facilitate the development of intricate relationships between different species. While many previous studies have focused on interspecies interactions within oral microbial communities (Palmer et al., 2001; Sharma et al., 2005; Wang and Kuramitsu, 2005; Kuramitsu et al., 2007; Saito et al., 2008), research on multispecies biofilms composed of soil bacteria is still in its infancy. In this study we assess, for the first time, the prevalence of interactions leading to synergism in biofilm formation. This was done by screening seven soil bacteria, isolated from one microhabitat, for biofilm formation in four-species cocultures.

In total, $63 \%$ of four-species biofilms showed synergistic effects in biofilm formation. This is in agreement with the assumption that the driving force in bacterial community development is self-organization and cooperation rather than competition of individual microorganisms (Davies et al., 1998; Daniels et al., 2004; Parsek and Greenberg, 2005). The recently presented 'Black Queen Hypothesis' provides an explanation as to how multispecies cooperation can evolve (Morris et al., 2012). This hypothesis considers cooperation in complex bacterial communities as being a consequence of species adapting to the presence of each other. In order to enhance individual fitness, species delete vital functions or pathways that are provided by the surrounding bacteria. This leads to a communal dependency and is, therefore, an irreversible commitment to living in close association with other species, which may often require development of more complex systems to ensure that the co-existence is maintained. When applying the Black Queen Hypothesis on the results of the present study, the strains may reside within the multispecies biofilm in order to keep vital partners in close contact, or alternatively, the ability of biofilm formation could be the function that is lost by some of the species.

Synergistic interactions were also observed when four poor biofilm-formers were cocultured (Figure 1b), verifying previous findings in which individual biofilm-forming ability is not necessarily an indicator of potential in multispecies biofilms (Burmølle et al., 2006; Bharathi et al., 2011). This shifting of biofilm pattern from weak to moderate or strong can be the result of metabolic interactions (Møller et al., 1998), enhanced coaggregation (Rickard et al., 2003), organized spatial distribution (Skillman et al., 1998) and/or facilitated initial surface attachment (Simões et al., 2008; Klayman et al., 2009), for example, bridging bacteria may facilitate the association of other species that do not coaggregate directly with each other. Thus, species that do not form biofilms as single strains may benefit from the advantages associated with biofilm formation, including enhanced protection from external stress and expanded niche availability, through engagement with multispecies communities.

Only two of the four-species cocultures tested showed a reduction in biofilm biomass in comparison with the best monospecies biofilm former present, indicating a low prevalence of strong competition. In contrast to our results, Foster and Bell, (2012) recently showed that the great majority of interactions in pairwise species combinations of bacterial isolates from tree-hole rainwater pools were net negative and very few strong higher-order positive effects arose from combinations composed of more than two species (Foster and Bell, 2012). The two studies differ with respect to bacterial habitats targeted for isolation (soil vs tree holes), productivity parameters assessed (biofilm formation vs $\mathrm{CO}_{2}$ production) and alternate definitions of synergism/cooperation. Such factors may explain the observed differences. The tree-hole species tend to use similar resources, which may be the key factor that leads to competition among microbes (Lawrence et al., 2012). While, in nutrient-rich agricultural soil, bacteria are likely to be tightly associated and individual bacterial consortia may stabilize their environment by their collective metabolic activities (Mathieu and Sonea, 1995). The key to whether bacterial species compete or cooperate may lie in their potential for long-term coadaptation and degree of niche overlap. We are currently investigating the significance of these parameters for the net interactions of bacterial communities.

\section{Enhanced biofilm formation in cocultures}

When exploring the strain dynamics of the selected four-species community, the strongest synergy was only induced when all the four strains 
(S. rhizophila, $X$. retroflexus, M. oxydans and $P$. amylolyticus) were cocultivated (Figure 2). This demonstrates that each strain had an important role in the enhanced biofilm formation irrespective of single-species biofilm formation ability. Divergence in resources use may be one factor leading to the increased productivity of the entire community. By differential resource consumption and metabolism of waste products produced by other species, the consortium collectively decomposes medium substrates more efficiently. In the present study, the synergistic enhancement of biomass of the four cocultured species was only observed when grown as a biofilm; planktonic coculturing did not lead to changes in overall biomass (data not shown) and we did not observe this synergistic effect when adding spent, cell-free supernatant to the three-species biofilms. This indicates that a structured environment and the physical presence of the interacting cells are highly important in maintaining synergy. Thus, syntrophy may explain the observed synergy, but only when coupled with a structured environment that enables tight cell-cell associations, optimizing product/substrate availability. Similar observations have previously been reported (Stewart et al., 1997; Hansen et al., 2007).

qPCR results showed a strong dominance (>97\%) of $X$. retroflexus in the four-species biofilm, which is consistent with the results from the CV assay where $X$. retroflexus was the only good biofilm former out of the four strains. Despite its monospecies biofilmforming abilities, cell numbers of strain $X$. retroflexus were enhanced approximately 60 -fold when cocultured in biofilms with the other strains. The remaining three strains constituted $<3 \%$ of the total cell number of the four-species biofilms; however, they all showed enhanced cell numbers in the multispecies biofilm by over three orders of magnitude when compared with cell numbers in monospecies biofilms. Thus, although S. rhizophila, $M$. oxydans and P. amylolyticus are present in low abundances and show poor capability of monospecies biofilm formation, they do stimulate biofilm formation of $X$. retroflexus and their cell numbers increase when present in the multispecies biofilm. This significant change in capacity of biofilm formation may be explained by the fact that species evolving in communities may have higher growth rates when assayed in the presence of other species (Lawrence et al., 2012), especially in structured communities (Stewart et al., 1997; Hansen et al., 2007). P. amylolyticus, the strain for which cell numbers received the greatest enhancement, has previously been reported to produce antibacterial agents with broad-spectrum activity against both Gram-negative and Gram-positive bacteria (DeCrescenzo Henriksen et al., 2007). However, in this study, this strain appeared to stimulate rather than inhibit growth of the other three strains and no biofilm-stimulating or -inhibitory effects of spent $P$. amylolyticus supernatants were observed. Further studies to identify the changes in gene expression patterns in this four-species biofilm will lead to a deeper understanding of the underlying molecular mechanisms of synergy in bacterial communities.

\section{Synergy in biofilm formation indicate collective cooperation}

Several observations in this study indicate that bacteria increase fitness from joining multispecies biofilms. If this fitness advantage applies to all of the species present, the underlying interaction is categorized as being cooperative (West et al., 2007). Four such observations are discussed here. (i) We observed a high prevalence of biofilm synergy in four-species biofilms. This implies selection for living in multispecies communities, indirectly indicating that bacteria may benefit from joining multispecies biofilms. The obtained fitness advantage may be caused by growth promotion that enhances bacterial biomass and thereby the direct fitness, but also the advantages gained from the biofilm-associated bacterial protection may be of major significance in natural high-stress environments. (ii) Each of the four species in the selected community was indispensable for the synergistic effect observed on biofilm biomass. These vital interdependencies may evolve over many years under continuous selective pressures, whereby only fitness-enhancing relationships are favored. (iii) The cell numbers of all four species were higher in multispecies biofilms than in their respective single-species biofilms. Thus, when focusing on biofilm-associated growth, there is a direct fitness advantage for all strains from joining the four-species biofilm. (iv) The observed synergistic effect only applies to the four-species biofilm; cell numbers in the planktonic fraction decreased for three out of the four strains. Based on this, there seem to be selection for, or forces that drive, bacteria to form multispecies biofilms. Alternatively, syntrophy occurs in the biofilm, whereby the growth rates of biofilm-resident strains are enhanced.

In conclusion, the observations presented in this study suggest multispecies biofilms as being a favorable bacterial habitat, in which protection and potential for engagement in mutually beneficial cooperative interactions prevail.

\section{Conflict of Interest}

The authors declare no conflict of interest.

\section{Acknowledgements}

We thank Timothy Evison and Urvish Travedi for text edition and Karin Vestberg and Anette Hørdum Løth for technical assistance. This study was partly funded by grants to SJS and $\mathrm{MB}$ from the Danish Innovation Consortium SiB, ref no: 11804520, and The Danish Council for Independent Research; ref no: DFF-133500071 and ref no: DFF-1323-00235 (SIMICOM). 


\section{References}

Bharathi P, Bhowmick P, Shekar M, Karunasagar I. (2011). Biofilm formation by pure and mixed culture of Lactobacillus isolates on polystyrene surface in varying nutrient conditions. Biotechnol Bioinf Bioeng 1: 93-98.

Burmølle M, Webb JS, Rao D, Hansen LH, Sørensen SJ, Kjelleberg S. (2006). Enhanced biofilm formation and increased resistance to antimicrobial agents and bacterial invasion are caused by synergistic interactions in multispecies biofilms. Appl Environ Microbiol 72: 3916-3923.

Burmølle M, Thomsen TR, Fazli M, Dige I, Christensen L, Homøe P. (2010). Biofilms in chronic infections -a matter of opportunity -monospecies biofilms in multispecies infections. FEMS Immunol Med Microbiol 59: 324-336.

Burmølle M, Kjøller A, Sørensen SJ. (2012). An invisible workforce: biofilms in the soil. In Microbial Biofilms-Current Research and Applications. Lear G, Lewis G (eds). Caister Academic Press: Norfolk, UK, pp 61-72.

Burmølle M, Ren D, Bjarnsholt T, Sørensen SJ. (2014). Interactions in multispecies biofilms: do they actually matter? Trends Microbiol 22: 84-91.

Ceri H, Olson M, Stremick C, Read R, Morck D, Buret A. (1999). The Calgary biofilm device: new technology for rapid determination of antibiotic susceptibilities of bacterial biofilms. J Clin Microbiol 37: 1771-1776.

Daniels R, Vanderleyden J, Michiels J. (2004). Quorum sensing and swarming migration in bacteria. FEMS Microbiol Rev 28: 261-289.

Davey ME, O’toole GA. (2000). Microbial biofilms: from ecology to molecular genetics. Microbiol Mol Biol Rev 64: 847-867.

Davies DG, Parsek MR, Pearson JP, Iglewski BH, Costerton J, Greenberg E. (1998). The involvement of cell-to-cell signals in the development of a bacterial biofilm. Science 280: 295-298.

de la Cruz Perera CI, Ren D, Blanchet M, Dendooven L, Marsch R, Sørensen SJ. (2013). The ability of soil bacteria to receive the conjugative IncP1 plasmid, pKJK10, is different in a mixed community compared to single strains. FEMS Microbiol Lett 338: 95-100.

DeCrescenzo Henriksen E, Phillips D, Peterson J. (2007). Polymyxin E production by $P$. amylolyticus. Lett Appl Microbiol 45: 491-496.

Diaz PI, Xie Z, Sobue T, Thompson A, Biyikoglu B, Ricker A. (2012). Synergistic interaction between Candida albicans and commensal oral streptococci in a novel in vitro mucosal model. Infect Immun 80: 620-632.

Filoche S, Anderson S, Sissons C. (2004). Biofilm growth of Lactobacillus species is promoted by Actinomyces species and Streptococcus mutans. Oral Microbiol Immunol 19: 322-326.

Foster KR, Bell T. (2012). Competition, not cooperation, dominates interactions among culturable microbial species. Curr Biol 22: 1845-1850.

Hall-Stoodley L, Costerton JW, Stoodley P. (2004). Bacterial biofilms: from the natural environment to infectious diseases. Nature Rev Microbiol 2: 95-108.

Hansen SK, Rainey PB, Haagensen JA, Molin S. (2007). Evolution of species interactions in a biofilm community. Nature 445: 533-536.
Jefferson KK. (2004). What drives bacteria to produce a biofilm? FEMS Microbiol Lett 236: 163-173.

Klayman BJ, Volden PA, Stewart PS, Camper AK. (2009). Escherichia coli O157: H7 requires colonizing partner to adhere and persist in a capillary flow cell. Environ Sci Technol 43: 2105-2111.

Kuramitsu HK, He X, Lux R, Anderson MH, Shi W. (2007). Interspecies interactions within oral microbial communities. Microbiol Mol Biol Rev 71: 653-670.

Lawrence D, Fiegna F, Behrends V, Bundy JG, Phillimore AB, Bell T. (2012). Species interactions alter evolutionary responses to a novel environment. PLoS Biol 10: e1001330.

Lee KWK, Periasamy S, Mukherjee M, Xie C, Kjelleberg S, Rice SA. (2014). Biofilm development and enhanced stress resistance of a model, mixed-species community biofilm. ISME J 8: 894-907.

Mathieu LG, Sonea S. (1995). A powerful bacterial world. Endeavour 19: 112-117.

Morris JJ, Lenski RE, Zinser ER. (2012). The Black Queen Hypothesis: evolution of dependencies through adaptive gene loss. mBio 3: e00036-12.

Møller S, Sternberg C, Andersen JB, Christensen BB, Ramos JL, Givskov M. (1998). In situ gene expression in mixed-culture biofilms: evidence of metabolic interactions between community members. Appl Environ Microbiol 64: 721-732.

Narisawa N, Haruta S, Arai H, Ishii M, Igarashi Y. (2008). Coexistence of antibiotic producing and antibioticsensitive bacteria in biofilms is mediated by resistant bacteria. Appl Environ Microbiol 74: 3887-3894.

Palmer RJ Jr, Kazmerzak K, Hansen MC, Kolenbrander PE. (2001). Mutualism versus independence: strategies of mixed-species oral biofilms in vitro using saliva as the sole nutrient source. Infect Immun 69: 5794-5804.

Parsek MR, Greenberg E. (2005). Sociomicrobiology: the connections between quorum sensing and biofilms. Trends Microbiol 13: 27-33.

Rao D, Webb JS, Kjelleberg S. (2005). Competitive interactions in mixed-species biofilms containing the marine bacterium Pseudoalteromonas tunicata. Appl Environ Microbiol 71: 1729-1736.

Ren D, Madsen J, Cruz-Perera C, Bergmark L, Sørensen S, Burmølle M. (2013). High-Throughput screening of multispecies biofilm formation and quantitative PCRbased assessment of individual species proportions, useful for exploring interspecific bacterial interactions. Microb Ecol, Doi:10.1007/s00248-013-0315-z.

Rickard AH, Gilbert P, High NJ, Kolenbrander PE, Handley PS. (2003). Bacterial coaggregation: an integral process in the development of multispecies biofilms. Trends Microbiol 11: 94-100.

Rodríguez SJ, Bishop PL. (2007). Three-dimensional quantification of soil biofilms using image analysis. Environ Eng Sci 24: 96-103.

Saito Y, Fujii R, Nakagawa KI, Kuramitsu H, Okuda K, Ishihara K. (2008). Stimulation of Fusobacterium nucleatum biofilm formation by Porphyromonas gingivalis. Oral Microbiol Immunol 23: 1-6.

Sharma A, Inagaki S, Sigurdson W, Kuramitsu H. (2005). Synergy between Tannerella forsythia and Fusobacterium nucleatum in biofilm formation. Oral Microbiol Immunol 20: 39-42.

Simões LC, Simoes M, Vieira MJ. (2008). Intergeneric coaggregation among drinking water bacteria: evidence of a role for Acinetobacter calcoaceticus as a bridging bacterium. Appl Environ Microbiol 74: 1259-1263. 
Skillman LC, Sutherland IW, Jones MV, Goulsbra A. (1998). Green fluorescent protein as a novel speciesspecific marker in enteric dual-species biofilms. Microbiology 144: 2095-2101.

Stewart PS, Camper AK, Handran S, Huang C-T, Warnecke M. (1997). Spatial distribution and coexistence of Klebsiella pneumoniae and Pseudomonas aeruginosa in biofilms. Microb Ecol 33: 2-10.

Sørensen SJ, Bailey M, Hansen LH, Kroer N, Wuertz S. (2005). Studying plasmid horizontal transfer in situ: a critical review. Nat Rev Microbiol 3: 700-710.
Wang BY, Kuramitsu HK. (2005). Interactions between oral bacteria: inhibition of Streptococcus mutans bacteriocin production by Streptococcus gordonii. Appl Environ Microbiol 71: 354-362.

West SA, Diggle SP, Buckling A, Gardner A, Griffin AS. (2007). The social lives of microbes. Annu Rev Ecol Evol Syst 38: 53-77.

Yamada M, Ikegami A, Kuramitsu HK. (2005). Synergistic biofilm formation by Treponema denticola and Porphyromonas gingivalis.. FEMS Microbio Lett 250: 271-277.

Supplementary Information accompanies this paper on The ISME Journal website (http://www.nature.com/ismej) 